\title{
A general correlation for the local loss coefficient in Newtonian axisymmetric sudden expansions
}

\author{
P.J. Oliveira ${ }^{\mathrm{a}, *}$, F.T. Pinho ${ }^{\mathrm{b}}$, A. Schulte ${ }^{\mathrm{c}}$ \\ a Departamento de Engenharia Electromecânica, Universidade da Beira Interior, Rua Marquês D’Ávila e Bolama, 6200 Covilhã, Portugal \\ b Departamento de Engenharia Mecânica e Gestão Industrial, Faculdade de Engenharia, Rua dos Bragas, 4099 Porto CODEX, Portugal \\ c Unidade de Térmica Industrial, INEGI-Instituto de Engenharia Mecânica e Gestão Industrial, Rua do Barroco, 175-4465 \\ S. Mamede de Infesta, Portugal
}

Received 25 November 1997; accepted 6 June 1998

\begin{abstract}
Results from numerical simulations and guidance from an approximated corrected-theory, developed by Oliveira and Pinho (1997), (Oliveira, P.J. and Pinho, F.T. 1997. Pressure drop coefficient of laminar Newtonian flow in axisymmetric sudden expansions. Int. J. Heat and Fluid flow 18, 518-529) have been used to arrive at a correlation expressing the irreversible loss coefficient for laminar Newtonian flow in axisymmetric sudden expansions. The correlation is valid for the ranges $1.5<D_{2} / D_{1}<4$ and $0.5<\operatorname{Re}<200$ with errors of less than $5 \%$, except for $25<\operatorname{Re}<100$ where the error could be as much as $7 \%$. The recirculation bubble length is also presented for the same range of conditions and the pressure recovery coefficient was calculated for Reynolds numbers above 15. (C) 1998 Elsevier Science Inc. All rights reserved.
\end{abstract}

Keywords: Sudden expansion; Loss coefficient; Pressure recovery coefficient; Laminar flow

\section{Notation}

$C_{\mathrm{I}}$

$\overline{C_{\mathrm{p} 01}}, \overline{C_{\mathrm{p} 02}}$

$C_{\mathrm{pM}}$

$D_{1}, D_{2}$

ER

$f_{x}, f_{y}$

$f_{1}, f_{2}$

$\left(f \equiv \Delta p_{\mathrm{F}} / \frac{L}{D} \frac{1}{2} \rho \bar{U}^{2}\right)$

$f_{2}^{\prime} \quad$ downstream friction coefficient for the real flow

$h \quad$ step height $h \equiv \frac{1}{2}\left(D_{2}-D_{1}\right)$

$L \quad$ pipe length

$L_{1}, L_{2} \quad$ inlet and outlet pipe lengths, respectively

$m_{\mathrm{i}} \quad$ coefficients of the fitting equations (Eq. (2))

$N \quad$ number of internal cells of computational grid (index letter refers to the direction, index number to the number of the block)

$p_{1}, p_{2} \quad$ pressure at inlet and outlet planes respectively

$\mathrm{Re} \quad$ Reynolds number $\left(\equiv \rho D_{1} \overline{U_{1}} / \mu\right)$

$\bar{U}_{1}, \bar{U}_{2} \quad$ bulk velocity in the inlet and outlet pipes

$\begin{array}{ll}x, y & \text { axial and radial coordinates } \\ \mu & \text { fluid dynamic viscosity; } \\ \Delta C_{\beta}, \Delta C_{\mathrm{F}}, & \text { corrective coefficients to standard theory (Oliveira } \\ \Delta C_{\mathrm{pO}} & \text { and Pinho, 1997); } \\ \Delta p & \text { total pressure drop }\left(\Delta p \equiv p_{1}-p_{2}\right) \\ \Delta p_{\mathrm{F}} & \text { fully developed pipe pressure drops } \\ & \left(\Delta p_{\mathrm{F}} \equiv \Delta p_{\mathrm{F}_{1}}+\Delta p_{\mathrm{F}_{2}}\right) \\ \Delta p_{\mathrm{R}} & \text { reversible pressure decrease, } \\ & \left(-\Delta p_{\mathrm{R}} \equiv \frac{1}{2} \rho U_{1}^{2}\left(1-\sigma^{2}\right)\right) \text { from Bernoulli equation; } \\ \sigma & \text { area ratio }\left(\equiv D_{1}^{2} / D_{2}^{2}\right) \\ \rho & \text { fluid density }\end{array}$

\section{Introduction}

Flow in sudden expansions is relevant in many applications in the chemical, pharmaceutical, food and biomedical industries, and its characteristics are documented in many references (Macagno and Hung, 1967; Back and Roshke, 1972; Pak et al., 1990). Often the experimental and numerical studies concentrate on the flow pattern features, like the size and strength of the recirculation zone and the existence of laminar or turbulent flow regimes (Halmos et al., 1975; Scott et al., 1986; Pak et al., 1990; Badekas and Knight, 1992).

In engineering calculations of piping networks it is the pressure drop and accurate prediction of the loss coefficients which are required. In many instances, either the pipe diameter is sufficiently small (as in hemodynamics) or the bulk velocity is sufficiently low (as with very viscous Newtonian and

\footnotetext{
${ }^{*}$ Corresponding author. E-mail: pjpo@ubi.pt.
} 
non-Newtonian fluids used e.g. in polymer processing) for the flow to remain laminar (typically for Reynolds numbers below 200, Macagno and Hung, 1967).

A theoretical expression for the irreversible loss coefficient, also known as the Borda-Carnot coefficient, $\left(C_{\mathrm{I}-\text { th }}\right)$ (with the irreversible loss coefficient defined as $C_{\mathrm{I}} \equiv\left(\Delta p-\Delta p_{\mathrm{R}}\right.$ $\left.-\Delta p_{\mathrm{F}}\right) /(1 / 2) \rho \bar{U}_{1}^{2}$, where $\Delta p_{\mathrm{R}}$ and $\Delta p_{\mathrm{F}}$ are the reversible and fully developed friction pressure drops, respectively, as defined in the notation) is available in the literature (Batchelor, 1967 ;Idel'cik, 1971); it is applicable to the turbulent flow of Newtonian fluids, but yields significant errors when applied at laminar, low Reynolds number flows as demonstrated by Oliveira and Pinho (1997). Based on overall quasi 1-D momentum and energy balances, these authors developed a corrected-theoretical expression for the loss coefficient $\left(C_{\text {Ith-c }}\right)$ in which the corrections to the standard expression $\left(C_{\mathrm{I}-\mathrm{th}}\right)$ accounted for three effects:

1. the velocity profile at the expansion plane deviates from the parabolic shape $\left(\Delta C_{\beta}\right)$;

2. the wall friction in the upstream pipe $\left(\Delta C_{\mathrm{F} 1}\right)$ and in the recirculation zone in the downstream pipe $\left(\Delta C_{\mathrm{F} 2}\right)$ deviates from the fully developed values;

3. the pressure variation at the expansion plane considerably deviates from the assumed uniform shape, particularly as the Reynolds number decreases to low values $\left(\Delta C_{\mathrm{p} 0}\right)$.

Thus Oliveira and Pinho wrote the loss coefficient as

$C_{\text {Ith-c }}=C_{\mathrm{I}-\text { th }}-\left(\Delta C_{\mathrm{F} 1}+\Delta C_{\mathrm{F} 2}+\Delta C_{\beta}-\Delta C_{\mathrm{p} 0}\right)$

and showed that this expression yields results in good agreement with careful full Navier-Stokes numerical simulations. The corrective terms in Eq. (1), however, were based on the numerical results and thus the method is not wholly predictive, still requiring empirical correlations to express $C_{\mathrm{I}}$. Nevertheless Oliveira and Pinho's approach is useful in highlighting which of the three effects above dominate the $C_{\mathrm{I}}$ expression at low $(\operatorname{Re}<10)$ and high $(\operatorname{Re}>10)$ Reynolds numbers, in the laminar regime, thus providing the means of choosing appropriate empirical correlations of $C_{\mathrm{I}}$ in terms of two parameters, the Reynolds number $(\mathrm{Re})$ and the expansion area ratio $(\sigma)$.

The purpose of the present note is to present numerical results of the loss coefficient over a wider range of conditions than that considered previously by Oliveira and Pinho (where $D_{2} / D_{1}=2.6$ ) and to derive a general correlation expressing $C_{\mathrm{I}}$ as a function of the Reynolds number and the expansion area ratio, for laminar Newtonian flow in axisymmetric sudden expansions. The pressure recovery coefficient $\left(C_{\mathrm{pM}}\right)$ is another relevant pressure-related quantity for the design of diffusers, and the detailed numerical work allowed its calculation at the higher range of laminar Reynolds numbers $(>10)$, for all expansion ratios.

The next section briefly outlines the numerical methodology, which follows closely the previous work. This is followed by the results of the numerical work and by the general $C_{\mathrm{I}}$ correlation for the sudden expansion.
Table 1

Mesh characteristics of the inlet pipe

\begin{tabular}{lllll}
\hline$L_{1} / D_{1}$ & $N_{x 1}$ & $N_{y 1}\left(=N_{y 2}\right)$ & $f_{x 1}$ & $f_{y 1}\left(=f_{y 2}\right)$ \\
\hline 40 & 48 & 20 & 0.8774 & 0.9236 \\
\hline
\end{tabular}

\section{Numerical method}

Oliveira and Pinho (1997) compared experimental and numerical data with their predictions and showed that their methodology was capable of correctly predicting the local loss coefficient.

In their calculations the Navier-Stokes equations were solved numerically by means of a finite-volume method using non-orthogonal collocated grids. The discretisation and interpolation methods were of second order accuracy making use of the linear upwind differencing scheme to approximate the first-order derivatives of the convective term and central differencing for the diffusion terms. Fig. 1 shows the general features of the computational domain and other details can be obtained in the original reference. The numerical results in Oliveira and Pinho (1997) pertained to a sudden expansion with a diameter ratio of $D_{2} / D_{1}=2.6$, and the objective here is to repeat the calculations at expansion diameter ratios of 1.5 , 2, 3 and 4, and to get other relevant quantities for the design of diffusers, of which the sudden expansion is a limiting case, such as the pressure recovery coefficient.

Some characteristics of the calculations and of the computational meshes are briefly commented particularly with attention to changes required by varying the larger, downstream pipe diameter. Previous mesh refinement studies showed that the main parameter controlling the precision of the results is the size of the smallest control-volume near the reentrant corner. Thus, the mesh is finer close to the sudden expansion plane and the upstream pipe wall, with the smallest normalised dimension, in both the axial and radial directions, equal to 0.01 .

A fully developed velocity profile was imposed at the inlet, which was far upstream of the sudden expansion plane, and a locally parabolic flow was assumed at the outlet (i.e. $\partial / \partial x=0)$. The flow was allowed to reattach and redevelop downstream of the sudden expansion, so the inlet and outlet pipe lengths had to be changed according to the expansion ratio and Reynolds number due to the different development lengths. Consequently, the number of computational cells in each direction also varied with those quantities in order to maintain the required mesh fineness. Tables 1 and 2 summarize the geometric and grid quantities for the various expansion ratios. In all cases, the length of the outlet pipe was kept at $L_{2}=60 D_{2}$, except for $D_{2} / D_{1}=4$ where a longer outlet pipe of $100 D_{2}$ was required to guarantee a fully developed flow over a significant portion of the outlet pipe. The calculations were carried out for Reynolds numbers ranging from 0.5 to 200 .

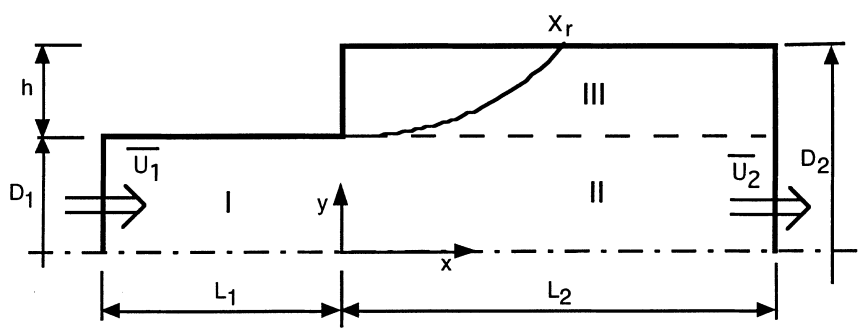

Fig. 1. Schematic representation of the sudden expansion and nomenclature. 
Table 2

Mesh characteristics of the outlet pipe for various expansion ratios

\begin{tabular}{lccccc}
\hline$D_{2} / D_{1}$ & $L_{2} / D_{1}$ & $N_{x 2 / 3}$ & $N_{y 3}$ & $f_{x 2 / 3}$ & $f_{y 3}$ \\
\hline 1.5 & 90 & 78 & 16 & 1.0888 & 1.0496 \\
2.0 & 120 & 82 & 20 & 1.0879 & 1.0827 \\
2.6 & 156 & 86 & 36 & 1.0868 & 1.0386 \\
3.0 & 180 & 86 & 36 & 1.0890 & 1.0498 \\
4.0 & 400 & 100 & 40 & 1.084 & 1.0564 \\
\hline
\end{tabular}

Table 3

Recirculation bubble length, $x_{\mathrm{R}} / h$

\begin{tabular}{rccccc}
\hline $\mathrm{Re}$ & $\mathrm{ER}=1.5$ & $\mathrm{ER}=2.0$ & $\mathrm{ER}=2.6$ & $\mathrm{ER}=3.0$ & $\mathrm{ER}=4.0$ \\
\hline 0.5 & 0.603 & 0.529 & 0.490 & 0.481 & 0.450 \\
1.0 & 0.615 & 0.549 & 0.510 & 0.505 & 0.475 \\
2.0 & 0.642 & 0.592 & 0.560 & 0.557 & 0.529 \\
3.5 & 0.689 & 0.664 & 0.650 & 0.645 & 0.621 \\
5.0 & 0.736 & 0.746 & 0.740 & 1.15 & 0.726 \\
10.0 & 0.926 & 1.07 & 1.11 & 1.14 \\
12.5 & 1.03 & 1.26 & 1.33 & 1.38 & 1.38 \\
17.5 & 1.24 & 1.65 & 1.79 & 2.87 & 1.89 \\
25 & 1.57 & 2.27 & 2.51 & 3.71 & 2.69 \\
35 & 2.03 & 3.12 & 3.51 & 5.33 & 3.79 \\
50 & 2.73 & 4.43 & 5.04 & 10.8 & 5.48 \\
100 & 5.20 & 8.87 & 10.2 & 16.3 & 11.1 \\
150 & 7.72 & 13.3 & 15.4 & 21.8 & 16.8 \\
200 & 10.3 & 17.8 & 20.5 & & 22.5 \\
\hline
\end{tabular}

Table 4

Pressure recovery coefficient, $C_{\mathrm{pM}}$

\begin{tabular}{rlllll}
\hline $\mathrm{Re}$ & $\mathrm{ER}=1.5$ & $\mathrm{ER}=2.0$ & $\mathrm{ER}=2.6$ & $\mathrm{ER}=3.0$ & $\mathrm{ER}=4.0$ \\
\hline 17.5 & 0.769 & 0.660 & 0.575 & 0.539 & 0.487 \\
25 & 0.476 & 0.474 & 0.373 & 0.309 & 0.295 \\
35 & 0.468 & 0.492 & 0.404 & 0.355 & 0.265 \\
50 & 0.480 & 0.509 & 0.435 & 0.379 & 0.277 \\
100 & 0.499 & 0.545 & 0.452 & 0.387 & 0.271 \\
150 & 0.508 & 0.552 & 0.452 & 0.384 & 0.264 \\
200 & 0.515 & 0.554 & 0.450 & 0.381 & 0.259 \\
\hline
\end{tabular}

\section{Results}

In the previous work (Oliveira and Pinho, 1997), predicted values of the recirculation length $\left(x_{\mathrm{R}}\right)$, eddy intensity and other quantities have been compared with experimental data and empirical correlations from the literature and generally there was good agreement hence validating the present numerical method. This exercise will not be repeated here but, for the sake of completeness, we give in Table 3 the predicted recirculation length normalised by the step height $\left(x_{\mathrm{R}} / h\right)$ for the various expansion ratios and Reynolds numbers. As an example of the agreement with results from other authors, the predicted recirculation lengths were within $2.5 \%$ of the values given by the correlation of Badekas and Knight (1992) in its range of validity $(50 \leqslant R e \leqslant 200$ and $1.5 \leqslant E \leqslant 6)$, except for $E=1.5$ where the differences were of the order of $20 \%$. However, the correlation of Badekas and Knight (1992) also has a large error for this expansion ratio, as documented by the authors themselves.

The values of the pressure recovery coefficient (defined as, $C_{\mathrm{pM}} \equiv\left(\bar{p}_{\mathrm{max}, 2}-\bar{p}_{01}\right) / \rho \bar{U}_{1}^{2}$, where $\bar{p}_{\mathrm{max}, 2}$ is the maximum areaaveraged pressure downstream of the expansion and $\bar{p}_{01}$ the value immediately upstream of the expansion plane) are listed in Table 4. The coefficient reaches a maximum for an expansion ratio of 2 , which is to be expected since for smaller expansion ratios the pressure recovery is overtaken by the frictional pressure drop (in the limit of a 1:1 expansion, the pressure continuosly drops due to the frictional losses). For all cases, the pressure recovery coefficient tends to stabilise with the Reynolds number.

The main emphasis of this note is on the loss coefficients $C_{\mathrm{I}}$, numerical calculations of which resulted in the values listed in Table 5 and plotted with marker symbols in Fig. 2 where the equations that were fitted to each set of values are also shown with lines. From an assessment of mesh refinement (see Oliveira and Pinho, 1997) and uncertainties related with slight changes in boundary and other conditions for the same flow ${ }^{1}$, the numerical error of the $C_{\mathrm{I}}$ predictions in Table 5 is estimated to be below $0.8 \%$. It can be seen in this figure that, as the expansion ratio increases, the $C_{\mathrm{I}}$ goes through a slight minimum value at intermediate Reynolds numbers. It is this

\footnotetext{
${ }^{1}$ Refers to pressure boundary conditions at solid walls and the presence or not of the $-(2 / 3) \mu \operatorname{div} \mathbf{V}$ term in the Newtonian constitutive equation.
} 
Table 5

$C_{\mathrm{I}}$ as a function of the expansion ratio $\left(\mathrm{ER}=D_{2} / D_{1}\right)$ and Reynolds number

\begin{tabular}{rccccc}
\hline $\mathrm{Re}$ & $\mathrm{ER}=1.5$ & $\mathrm{ER}=2.0$ & $\mathrm{ER}=2.6$ & $\mathrm{ER}=3.0$ & $\mathrm{ER}=4.0$ \\
\hline 0.5 & 19.58 & 28.83 & 33.00 & 34.28 & 35.71 \\
1.0 & 9.800 & 14.42 & 16.52 & 17.16 & 8.622 \\
2.0 & 4.915 & 7.246 & 8.330 & 4.999 & 8.983 \\
3.5 & 2.837 & 4.192 & 4.810 & 3.586 & 5.213 \\
5.0 & 2.018 & 2.994 & 3.445 & 2.079 & 3.744 \\
10.0 & 1.101 & 1.683 & 1.978 & 1.839 & 2.200 \\
12.5 & 0.931 & 1.456 & 1.733 & 1.621 & 1.966 \\
17.5 & 0.752 & 1.229 & 1.514 & 1.508 & 1.764 \\
25 & 0.633 & 1.093 & 1.383 & 1.460 & 1.668 \\
35 & 0.564 & 1.025 & 1.335 & 1.441 & 1.633 \\
50 & 0.521 & 0.987 & 1.310 & 1.443 & 1.625 \\
100 & 0.485 & 0.965 & 1.304 & 1.451 & 1.641 \\
150 & 0.476 & 0.963 & 1.309 & 1.458 & 1.653 \\
200 & 0.475 & 0.966 & & & 1.662 \\
\hline
\end{tabular}

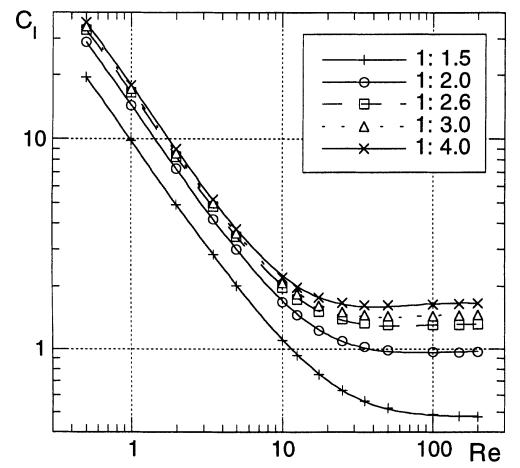

Fig. 2. Comparison between the calculated local loss coefficient (symbols) and the fitted correlation (lines) Eq. (2) for various sudden expansion ratios.

intermediate region that brings about some difficulties in fitting with good accuracy a general curve to the data.

The fitted equations, plotted as solid and broken lines in Fig. 2, were guided by the theoretical work in Oliveira and Pinho (1997), who derived a correlation for the case of the 2.6 expansion ratio of the form

$C_{\mathrm{I}}=\frac{m_{1}}{\mathrm{Re}^{m_{2}}}+m_{3}+m_{4} \log \mathrm{Re}+m_{5}(\log \mathrm{Re})^{2}$.

The first term on the right-hand side of Eq. (2) fits the lowest Reynolds number data, where the corrective term $\Delta C_{\mathrm{p} 0}$ is dominant, as mentioned in the introduction. In the approximated theory of Oliveira and Pinho, this corrective term was given by

$\Delta C_{\mathrm{p} 0}=(1-\sigma)\left(\overline{C_{\mathrm{p} 01}}-\overline{C_{\mathrm{p} 02}}\right)$

and the difference between the pressure coefficients just upstream $\left(\overline{C_{\mathrm{p} 01}}\right)$ and downstream $\left(\overline{C_{\mathrm{p} 02}}\right)$ from the expansion plane was observed to vary as $\mathrm{Re}^{-1}$, but showed no explicit dependence on $\sigma$. This trend is precisely reflected in the first term in Eq. (2), with $m_{2} \approx 1$ (a mild function of $\sigma$ ) and $m_{1} \approx 30((24 / 30)-\sigma)$ from Table 6 .

The independent and linear log terms were necessary to fit the data at the highest Reynolds numbers where $C_{\mathrm{I}}$ is controlled by the constant $C_{\mathrm{I} \text {-th }}$ term and the wall friction corrective term $\Delta C_{\mathrm{F} 2}$. Since $C_{\mathrm{I}-\mathrm{th}}=2(1-\sigma)(1-(1 / 3) \sigma)$ (for parabolic velocity profiles) and $\Delta C_{\mathrm{F} 2} \equiv \sigma^{2}\left(L_{2} / D_{2}\right)\left(f_{2}-f_{2}^{\prime}\right)$ $\propto \sqrt{\sigma}$ we end up with a complex polynomial dependence of $C_{\mathrm{I}}$ on $\sigma$, as reflected in the form of the $m_{3}$ function (cf. Table 6).
The final term ensured a good fitting at the intermediate range of Reynolds numbers. For each expansion ratio, small deviations between the data and each individual corresponding correlation, were apparent only at intermediate Reynolds numbers and for the higher expansion ratios (see Fig. 2), but they never exceed $2.5 \%$.

In order to build a single equation for all expansions the individual $m_{\mathrm{i}}$ coefficients of Eq. (2) were correlated with the expansion area ratio $\left(\sigma=A_{1} / A_{2}=\left(D_{1} / D_{2}\right)^{2}\right)$. The symbols in Fig. 3 show the monotonic variations of the $m_{\mathrm{i}}$ coefficients with $\sigma$. The coefficients could be fitted by appropriate equations using the algorithm of Press et al. (1992). Some could be fitted by either a 2 nd-order-polynomial or by an exponential function, both leading to a similar degree of uncertainty, but the latter were preferred for simplicity. Table 6 gives the appropriate functions for the five coefficients.

After combining the selected equations from Table 6 with Eq. (1) the following general expression for the local loss coefficient was obtained

$$
\begin{aligned}
C_{\mathrm{I}}= & \frac{24.044-30.42 \cdot \sigma}{\operatorname{Re}^{0.88522+0.29043 \cdot \sigma-0.25408 \cdot \sigma^{2}}}-5.761 \mathrm{e}^{-4.5284 \cdot \sigma} \\
& +6.2933 \mathrm{e}^{-4.3898 \cdot \sigma} \log \mathrm{Re} \\
& -1.3023 \mathrm{e}^{-4.6663 \cdot \sigma}(\log \mathrm{Re})^{2} .
\end{aligned}
$$

The maximum deviation between this general correlation and the numerical $C_{\mathrm{I}}$ data is less than $7 \%$, as can be checked in Fig. 4. Further refinements of Eq. (2) were carried out to reduce the number of digits and resulted in

Table 6

Equations fitted to the coefficients $m_{1}$ through $m_{5}$ of Eq. (2)

$$
\begin{aligned}
& m_{1}=24.044-30.42 \sigma \\
& m_{2}=0.88522+0.29043 \sigma-0.25408 \sigma^{2} \\
& m_{3}=-5.2938+18.117 \sigma-17.826 \sigma^{2} \\
& \text { or } \\
& m_{3}=-5.761 \mathrm{e}^{-4.5284 \sigma} \\
& m_{4}=5.7946-19.289 \sigma+18.545 \sigma^{2} \\
& \text { or } \\
& m_{4}=6.2933 \mathrm{e}^{-4.3898 \sigma} \\
& m_{5}=-1.1933+4.186 \sigma-4.1994 \sigma^{2} \\
& \text { or } \\
& m_{5}=-1.3023 \mathrm{e}^{-4.6663 \sigma}
\end{aligned}
$$



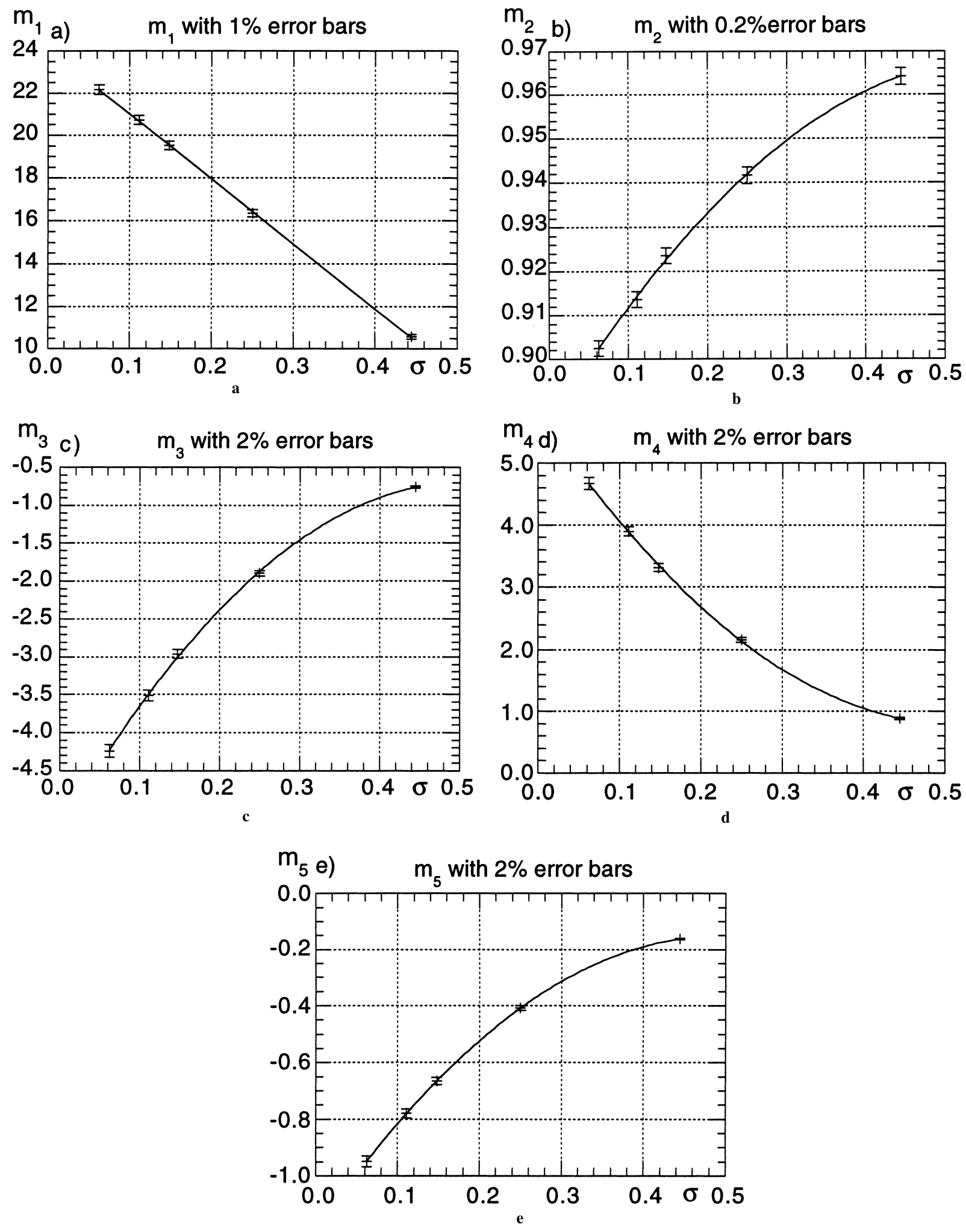

Fig. 3. Variation of the coefficients $m_{1}-m_{5}$ with the area ratio, $\sigma$.

$$
\begin{aligned}
C_{\mathrm{I}}= & \frac{24-30 \cdot \sigma}{\mathrm{Re}^{0.89+0.29 \cdot \sigma-0.25 \cdot \sigma^{2}}}-5.8 \mathrm{e}^{-4.5 \cdot \sigma} \\
& +6.3 \mathrm{e}^{-4.4 \cdot \sigma} \log \mathrm{Re}-1.3 \mathrm{e}^{-4.7 \cdot \sigma}(\log \mathrm{Re})^{2}
\end{aligned}
$$

for which the maximum error relative to the numerical data is still kept below 7\%.

Fig. 2 also shows that the loss coefficient is approximately constant, for a given expansion ratio, when the Reynolds 

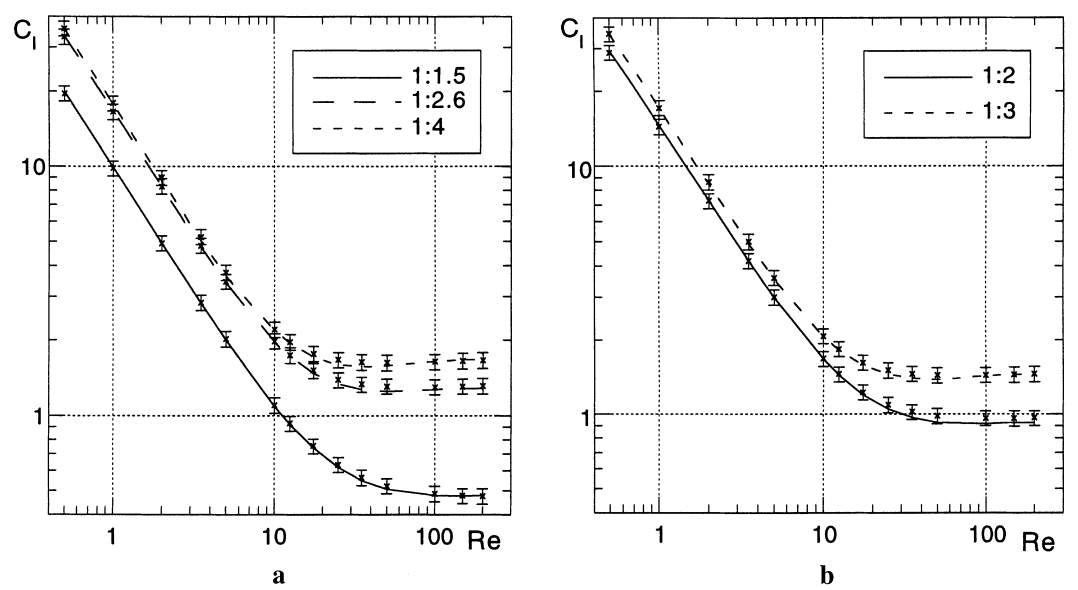

Fig. 4. Comparison between the calculated local loss coefficient $(\times)$ and Eq. (5) (lines), with 7\% error bars as a function of the Reynolds number and expansion ratio.

Table 7

Values of $C_{\mathrm{I}} /(1-\sigma)^{2}$ for $\operatorname{Re} \geqslant 50$ and $1.5 \leqslant \sigma \leqslant 4$ (Eq. (6))

\begin{tabular}{|c|c|c|c|c|c|}
\hline ER & 1.5 & 2.0 & 2.6 & 3.0 & 4.0 \\
\hline$K$ & 1.54 & 1.72 & 1.80 & 1.83 & 1.88 \\
\hline
\end{tabular}

number is greater than approximately 50 (the high Reynolds number range under laminar flow conditions). The uncorrected theory gives $C_{\mathrm{I}-\mathrm{th}}=(1-\sigma)^{2}$ for uniform velocity profiles and $C_{\text {I-th }}=2(1-\sigma)(1-(1 / 3) \sigma)$ for parabolic velocity profiles; the actual predicted values at high Reynolds numbers in Table 5 fall in between those bounds but closer to the latter, as it would be expected. Still, we can correlate the local loss coefficient as

$C_{\mathrm{I}}=K(1-\sigma)^{2}$

valid for $\operatorname{Re} \geqslant 50$ and $1.5 \leqslant \sigma \leqslant 4$, with $K$ given in Table 7 . The maximum error of this correlation in this range is $2.0 \%$, except for $\sigma=1.5$ where the error can be as high as $9 \%$ close to the lower limit of the Reynolds number.

\section{Conclusions}

Results from numerical simulations and guidance from an approximated corrected theory, developed in a previous work, have been used to arrive at an empirical formula expressing the irreversible loss coefficient for laminar Newtonian flow in axisymmetric sudden expansions. The proposed correlation is given by Eq. (5), which is a best fit for the ranges $1.5<D_{2} /$ $D_{1}<4$ and $0.5<\operatorname{Re}<200$ with errors of less than $5 \%$, except for $25<\operatorname{Re}<100$ where the error could be as much as $7 \%$.

Predicted values of the recirculation length and of the pressure recovery coefficient are also given in tabulated form since this information may be useful for future validation studies and for engineering design of diffusers.

\section{Acknowledgements}

This work was in part supported by Junta Nacional de Investigação Científica e Tecnológica, Portugal, under project PBIC/C/QUI/1980. A. Schulte acknowledges the financial support of the EC programme Leonardo da Vinci. The authors are listed alphabetically.

\section{References}

Back, L., Roshke, E., 1972. Shear layer regimes and wave instabilities and reattachment lengths downstream of an abrupt circular channel expansion. J. Appl. Mech. 677-681.

Badekas, D., Knight, D.D., 1992. Eddy correlations for laminar axisymmetric sudden expansion flows. J. Fluids Eng. 114, 119-121.

Batchelor, G.K., 1967. An Introduction to Fluid Dynamics. Cambridge University Press, Cambridge.

Halmos, A.L., Boger, D.V., Cabelli, A., 1975. The behaviour of a power law fluid flowing through a sudden expansion. Part II. Experimental verification. 21, 540-549.

Idel'cik, I.E., 1971. Memento des Pertes de Charge. Editions Eyrolles, Paris.

Macagno, E.O., Hung, T.K., 1967. Computation and experimental study of a captive annular eddy. J. Fluid Mech. 28, 43-64.

Oliveira, P.J., Pinho, F.T., 1997. Pressure drop coefficient of laminar Newtonian flow in axisymmetric sudden expansions. Int. J. Heat and Fluid Flow 18, 518-529.

Pak, B., Cho, Y.I., Choi, S.U.S., 1990. Separation and reattachment of non-Newtonian fluid flows in a sudden expansion pipe. J. NonNewt. Fluid Mech. 37, 175-199.

Press, W.H., Flannery, B.P., Teukolsky, S.A., Vetterling, W.T., 1992. Numerical Recipes in Fortran, 2nd ed., Cambridge University Press, Cambridge.

Scott, P., Mirza, F., Vlachopoulos, J., 1986. A finite element analysis of laminar flows through planar and axisymmetric abrupt expansions. Computers and Fluids 14, 423-432. 